\title{
Carole Edwards (dir.), Le sacrifice dans les littératures francophones
}

\section{Carminella Biondi}

\section{Q OpenEdition \\ 1 Journals}

\section{Edizione digitale}

URL: http://journals.openedition.org/studifrancesi/640

DOI: 10.4000/studifrancesi.640

ISSN: 2421-5856

\section{Editore}

Rosenberg \& Sellier

\section{Edizione cartacea}

Data di pubblicazione: 1 aprile 2015

Paginazione: 202-203

ISSN: 0039-2944

\section{Notizia bibliografica digitale}

Carminella Biondi, «Carole Edwards (dir.), Le sacrifice dans les littératures francophones », Studi Francesi [Online], 175 (LIX | I) | 2015, online dal 01 avril 2015, consultato il 18 septembre 2020. URL : http:// journals.openedition.org/studifrancesi/640; DOI : https://doi.org/10.4000/studifrancesi.640

Questo documento è stato generato automaticamente il 18 settembre 2020.

\section{(c) (i) $\odot$}

Studi Francesi è distribuita con Licenza Creative Commons Attribuzione - Non commerciale - Non opere derivate 4.0 Internazionale. 


\title{
Carole Edwards (dir.), Le sacrifice dans les littératures francophones
}

\author{
Carminella Biondi
}

\section{NOTIZIA}

CAROLE EDWARDS (dir.), Le sacrifice dans les littératures francophones, Amsterdam-New York, Rodopi, 2014, «Francopolyphonies», pp. 193.

1 Sin dall'incipit della sua introduzione, Carole Edwards dichiara una delle finalità maggiori del suo impegno nel progettare e coordinare questo lavoro attorno a un tema così complesso e così polisemico come quello del sacrificio. Si è proposta di stimolare la nascita di un lavoro collettivo che fosse in grado di dare una valida risposta a tutti coloro «qui perçoivent la francophonie comme une discipline quelque peu farfelue et surtout superficielle qui ressasserait les mêmes thèmes et inquiétudes à savoir le malaise identitaire, l'entre-deux, la douleur, la violence et surtout la difficulté de se fonder sur une culture propre postérieure au colonialisme. En somme, un atavisme francophone peu flatteur, rébarbatif et qui, en outre, doit faire ses preuves parmi les "Belles Lettres"» (p. 9). Indubbiamente la sintesi del concetto di francofonia fatta da Carole Edwards è, crediamo, volutamente un po' riduttiva, ma è indubbio che ha posto l'accento sui limiti della disciplina, in particolare per quanto concerne gli studi francofoni, perché gli scrittori mi sembra abbiano superato da tempo questa visione restrittiva e si siano mossi in territori aperti: basti pensare, per tutti, al pensiero e alla narrativa di Édouard Glissant, o ad una posizione, sia pure discutibile e discussa, come quella della «letteratura-mondo».

Questo non basta a spiegare la scelta del tema, che trova invece la sua motivazione in una visita in Senegal e all'isola di Gorea, luoghi-simbolo del più grande sacrificio di esseri umani di tutti i tempi, quello della tratta negriera, messa in atto per fornire manodopera gratuita al servizio dello sfruttamento del continente americano. Naturalmente questo è solo il punto di partenza, una dedica ideale al sacrificio per 
antonomasia, perché oggetto di questa raccolta di saggi non sono i fatti storici, ma i prodotti dell'arte e della letteratura: «Ce volume aborde donc l'étude plurivoque du sacrifice, qu'il s'applique au théâtre, au roman ou au film; qu'il vienne de l'Afrique Subsaharienne, de l'Afrique du Nord ou de la Caraïbe» (p. 10). Come appare evidente da questa breve sintesi, lo spazio indagato è quello della colonizzazione francese con popolazione africana o di origine africana, mentre la plurivocità degli apporti concerne sia la diversità di genere, letterario e genetico, sia il concetto di sacrificio, che può essere reale o simbolico, dono di sé o imposizione violenta, può concernere la storia raccontata o colui che la racconta, ecc.

3 Il volume riunisce otto interventi e si conclude, come ormai è consuetudine, con i résumés degli articoli e delle brevi biografie degli autori, ma contiene anche un utile, e non scontato, indice dei nomi. Sullo sfondo di quasi tutti i saggi, inevitabilmente, il lavoro del 1972 di René Girard, La violence et le sacré, particolarmente presente nel saggio d'apertura, di carattere più generale, di Abderrahmane BAIBECHE, La révolte entre la mort de Dieu et la mort de l'auteur (pp. 23-38), che ricostruisce il dibattito generale attorno al tema. Si passa poi alla rappresentazione di alcuni aspetti specifici del sacrificio, dapprima sul territorio africano poi antillano, in cui si evidenzia, sul piano generale, una concomitanza abbastanza frequente fra sacrificio, morte e follia. In alcuni casi il rapporto fra sacrificio e follia emerge fin dal titolo, come nel saggio di Gladys M. FRANCIS, Folies sacrificielles dans le théâtre francophone africain (pp. 39-63), che analizza in particolare quattro pièces: Qui a mangé Madame d'Avoine Bergotha (1989) e Antoine m'a vendu son destin (1997) del congolese Sony Labou Tansi, La légende de Wagadu vue par Sia Yataéré (1990) del mauriziano Moussa Diagana e Village fou ou les déconnards (2000) dell'ivoriano Koffi Kwahulé. Qui la follia è percepita come l'ingrediente-base di un'alienazione che porta al suicidio e che concerne nello specifico i popoli africani per poi dilatarsi fino ad abbracciare l'umanità. Alla scrittura è affidato il ruolo salvifico della liberazione dal male. Segue un saggio di una nota studiosa del teatro, e in particolare del teatro postcoloniale, Sylvie CHALAYE, che approfondisce l'opera di uno dei drammaturghi già oggetto di analisi nel lavoro precedente: Sacrifice et eucharistie dans le théâtre de Koffi Kwahulé (pp. 65-86), mettendo in evidenza la presenza della Bibbia nell'opera drammatica dello scrittore e la sua volontà di trasformare il sacrifico in offerta di una «diversità positiva», in vista di un futuro rapporto armonioso fra i popoli. Si resta in Africa anche con il terzo saggio della curatrice del volume, Carole EDWARDS, Tuer/Tu es: Patrice Lumumba et le Congo (pp. 75-86), che esamina il film Lumumba realizzato nel 2001 da Raoul Peck, da cui emerge che il sacrificio di Lumumba non ha prodotto l'attesa riconciliazione del Congo ma, trasformando il personaggio in mito, ha contribuito a porre le basi di una storia e di una cultura nate dalle rovine della colonizzazione: «Le sort de la violence et du sacrifice n'aboutit jamais, n'unit pas mais au contraire divise. Le rêve de Lumumba demeure pourtant utopique et s'étend aux autres nations africaines, voire au monde tout entier» (p. 84).

4 Si passa alle Antille con il saggio di Axel ARTHÉRON, Le Théâtre caribéen d'expression française: "La Tragédie du Roi Christophe" (Aimé Césaire), "Monsieur Toussaint" (Édouard Glissant) et "Dessalines ou la passion de l'indépendance" (Vincent Placoly) (pp. 87-106), che studia la rappresentazione teatrale del sacrificio dei padri della nazione haitiana da parte di alcuni dei grandi scrittori dell'arcipelago caraibico. Pur tenendo debitamente conto della diversità dei personaggi storici, dei rispettivi sacrifici e degli scrittori che li hanno riportati in vita, l'autore trae una conclusione che li accomuna: «L'Histoire de ce 
sacrifice paraît fournir les éléments nécessaires à la mise en forme d'une expression tragique, susceptible de dévoiler les pans cachés de sociétés antillaises encore à la recherche de leur équilibre» (p. 105). In "Cœur d'ébène" di Roland Brival: Victimes et/ou Martyrs, Maîtres et/ou Esclaves (pp. 108-119), Yolande HELM affronta in particolare il tema del meticciato, nell'opera dell'artista martinicano (oltre ad essere scrittore, Brival è anche pittore e musicista), perché secondo l'ammissione dello stesso Brival, in un paese in cui convivono popoli di origine diversa, il colore è importante. In epoca di creolizzazione, in cui la miscidanza è vissuta, o dovrebbe essere vissuta, come un fatto positivo, i personaggi di Brival, alla ricerca delle origini e della loro identità vivono ancora negativamente la loro incerta appartenenza: «cette quête est d'autant plus tourmentée pour l'homme métis écartelé entre l'Ancien et le Nouveau Monde et symbole de la scène maudite, du viol primordial» (p. 115). Nel saggio che segue, di Yolaine PARISOT, Sacrifice de l'auteur, désacralisation de l'écrivain-monde: l'exemple haïtien (pp. 121-141), come indica il titolo, il sacrificio non concerne il soggetto o il tema dell'opera, ma l'autore: «De fait la consécration scellée par les prix littéraires et par le Manifeste "Pour une littérature-monde en français" a pour corollaire la désacralisation du littéraire et d'une auctorialité toujours sujette à caution» (p. 137). Chiude il volume il saggio di Mylène DORCÉ, "Kenbé réd, pa lagé!" Dictature, colère, humour chez trois écrivaines haitiennes (pp. 143-167), consacrato a tre scrittrici haitiane, Marie Chauvet, MarieThérèse Colimon-Hall et Marie-Célie Agnant, che raccontano storie di donne oppresse sotto il governo di François e Jean-Claude Duvalier, ma oppresse anche dal regime familiare, che spesso ne richiede il sacrificio per poi prenderne le distanze. La scrittura diventa allora lo strumento che permette di «exorciser la souffrance de personnages meurtris par la vie et victimes de la cruauté des dirigeants haïtiens» (p. 165).

5 Non so se il volume sia davvero riuscito a orientare gli studi francofoni verso problematiche nuove, perché, in fondo, il tema identitario resta cruciale in molti degli autori analizzati e nei saggi critici, ma si tratta comunque di un lavoro importante, in cui l'elemento davvero nuovo è che si è ormai totalmente usciti dalla dialettica colonizzato e colonizzatore, che appare talvolta ancora sullo sfondo del racconto di questi popoli che stanno ormai facendo i conti con la loro storia. 